\title{
Impact of pump depletion on the determination of the Brillouin gain frequency in distributed fiber sensors
}

\author{
Luc Thévenaz , Stella Foaleng Mafang and Jie Lin ${ }^{\mathrm{a}}$ \\ EPFL Swiss Federal Institute of Technology, Group for Fibre Optics, Institute of Electrical Engineering, \\ STI IEL GR-SCI Station11, CH-1015 Lausanne, Switzerland \\ ${ }^{a}$ Permanent address: Bucknell University, Dept. of Electrical Engineering, Lewisburg PA 17837, USA
}

\begin{abstract}
The energy transfer between the two interacting optical waves in a distributed sensor based on stimulated Brillouin scattering can lead to a non-uniform spectral distribution of the pumping power after a long propagation. This results in a spectrally distorted gain that biases the determination of the maximum gain frequency. A quantitative analytical model gives an expression for the tolerable pump power change keeping the maximum bias within a given accuracy.
\end{abstract}

Keywords: Fiber optics, Optical fiber sensors, distributed fiber sensor, stimulated Brillouin scattering, Nonlinear optics.

\section{INTRODUCTION}

Distributed Brillouin fiber sensors have proved their capabilities to realize distributed measurements of temperature and strain over several tens of kilometers with a meter spatial resolution since the first demonstration in the late $1980^{\prime}{ }^{1}$. They are now considered as a maturing technology with recent progresses towards centimeter spatial resolution ${ }^{2}$ and over an extended distance range that is gradually reaching $100 \mathrm{~km}^{3,4,5}$.

The increased distance range raises new challenges caused by effects that have a negligible impact on short distances, but build up and turn extremely detrimental over long distances. Among these effects, the spectral transfers due to stimulated Raman scattering ${ }^{6}$ and modulation instability ${ }^{7}$ were identified several years ago. These two effects lead to a total extinction of the pump wave and an evidently observable absence of Brillouin gain after a critical distance directly scaled by the peak pump power. The other effect resulting from the gradual power transfer from the higher frequency wave to the lower frequency wave has not been considered so far as a major concern by the community since it is more insidious and does not prevent the basic operation of the sensor. However, its detrimental impact has been rightfully mentioned at a relatively early stage during the development of Brillouin sensing ${ }^{8,9}$. As a result of the increased range and accuracy, some recent reports have clearly focused on this effect by proposing a correction procedure based on an algorithm ${ }^{10}$, by explicitly checking its absence of negative impact through a proper scaling of the signal powers ${ }^{3,5}$ or by showing and evaluating experimentally its biasing effect ${ }^{11}$.

We propose in this paper to further develop and complete the work performed in Ref. $8 \& 9$ by building a full model that addresses the impact of the power transfer between the pump and the signal in the case of a BOTDA sensor. The analytical model not only can be used to evaluate the error on the determination of the maximum gain frequency in a given experimental configuration, but also defines the worst case condition with the maximal error. In addition, this model reveals the tolerable power transfer for a given maximum error. In the situation addressed by the model, a pulse and a CW wave counterpropagate in the fiber and interact through stimulated Brillouin scattering. The fiber sensor is defined to be in a gain configuration if the pulse frequency is higher than the $\mathrm{CW}$ wave frequency, otherwise in a loss configuration. The tiny $\mathrm{CW}$ wave intensity change observed in the time domain gives a measure of the interaction intensity at the crossing point of the pulse and the $\mathrm{CW}$ wave. This distributed intensity change actually is entirely compensated by an equivalent cumulated energy change in the pulse wave. The effect on the pulse amplitude can be quite massive with two identical power waves interacting over a long length, so that a clever design always employs an intense pump pulse and a weak CW wave to minimize the relative impact on the pulse amplitude. A significant change of the pulse power is detrimental because this power not only scales the strength of the interaction but also affects the response at the far end of the fiber. More concretely the output pulse power varies when the frequency difference between the two interacting waves is scanned. The variation inevitably leads to a spectral distortion of the gain spectrum

* luc.thevenaz@epfl.ch; phone +41 21693 4774; http://people.epfl.ch/luc.thevenaz

21st International Conference on Optical Fiber Sensors, edited by Wojtek J. Bock, Jacques Albert, Xiaoyi Bao, Proc. of SPIE Vol. 7753, 775322 - (c) 2011 SPIE · CCC code: 0277-786X/11/\$18 - doi: 10.1117/12.885084 


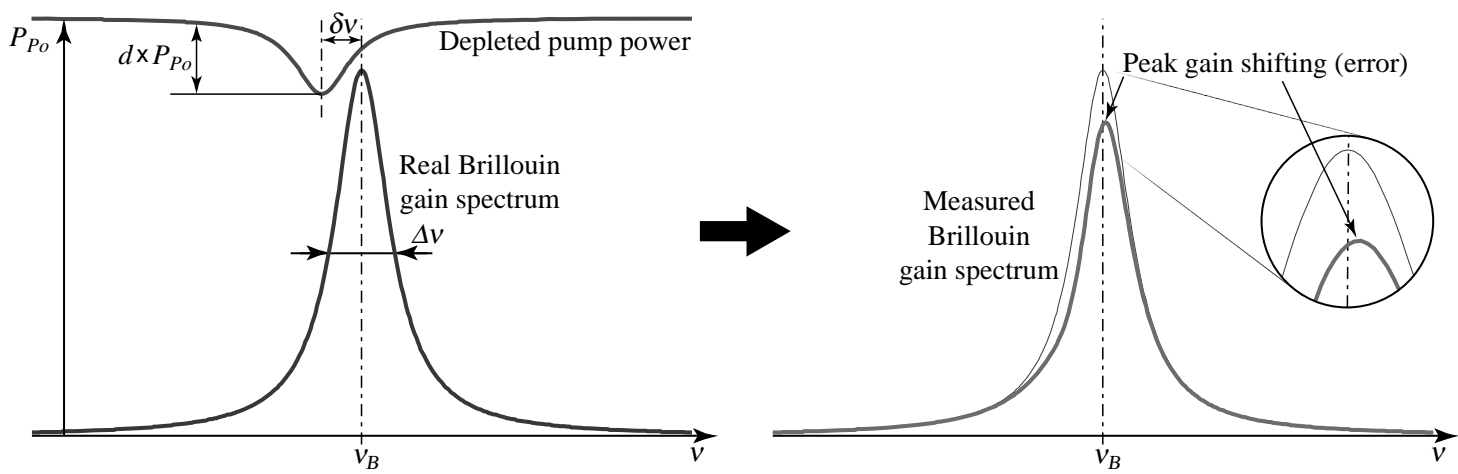

Fig.1: After propagation in a long uniform fiber a too strong CW signal will deplete the pump that will show a power drop when the frequency difference between pump and signal correspond to the Brillouin shift of this long fiber, following a power distribution as shown on the top left curve. If such a pump interrogates a segment with a gain spectrum shifted by a small amount $\delta v$, as shown on left, the actual measured gain spectrum shows a distorted skew distribution with a slightly shifted peak position, as shown on right. The thin line represents the real gain spectrum.

at a given position. It must be mentioned that both the gain and the loss configurations lead to an equally distorted gain spectrum, so that none of these configurations can be claimed to offer a decisive advantage. This conclusion is fully supported by the results of our model, so we shall address hereafter the case of a gain configuration without loss of generality. The power transfer from the pulse to the $\mathrm{CW}$ wave will now be designated as pump depletion. The pump pulse is chosen to be launched at the near end of the fiber $(z=0)$, the CW wave entering at the far end $(z=L)$.

The common sense shows that the worst case is the situation of a uniform fiber showing a constant Brillouin shift, albeit presenting a short section at the far end with different environmental conditions ${ }^{9,11}$, so that the cumulated depletion effect on the pulse is maximized at the maximum gain frequency. If ever the short section at the far end presents a gain spectrum that is slightly shifted with respect to the long uniform preceding section, it will be spectrally scanned by a pump with a frequency varying power as shown in Fig. 1. This results in a measured skew spectrum in the short section and the determination of the peak gain frequency will be biased. In this paper we shall only consider this worst case, all other situations leading to a less severe error.

\section{RELATION BETWEEN SIGNAL POWER AND DEPLETION}

As depicted in Fig. 1, the amount of depletion can be characterized by a dimensionless coefficient $d$ :

$$
d=\left(P_{P o}-P_{P}\right) / P_{P o}
$$

where $P_{P_{0}}$ is the pump power in absence of Brillouin interaction (absence of gain or no signal wave) and $P_{P}$ is the pump power in presence of maximum interaction at the Brillouin peak gain frequency. Assuming the effect of the interaction has negligible impact on the power $P_{S}$ of the CW signal wave (small gain condition), the signal launched at the far end at $z=L$ will only experience an exponential decay due to the linear attenuation $\alpha$, so that $P_{S}(z)=P_{i S} \mathrm{e}^{-\alpha(L-z)}$ with $P_{i S}=P_{S}(L)$ as the input signal power. Then the pump power distribution $P_{P}(z)$ can be calculated by solving the basic equation for the Brillouin interaction, including the linear attenuation term:

$$
d P_{P}=-\alpha P_{P}(z) d z-\frac{g_{B}}{A_{e f f}} P_{P}(z) P_{S}(z) d z
$$

where $g_{B}$ and $A_{\text {eff }}$ are the Brillouin linear gain and the nonlinear effective area of the propagating mode, respectively. It must be pointed out that $g_{B}$ is considered here as position-independent, since the maximal depletion effect will be observed in the worst case scenario when the gain is maximal at any position.

By inserting Eq.(1) into Eq.(2), an exact solution $P_{P}(z)$ can be found under the small gain assumption, giving the following expression for the residual output pump power $P_{P}(L)$ after fixing the initial condition $P_{i P}=P_{P}(0)$ :

$$
P_{P}(L)=P_{i P} \mathrm{e}^{-\alpha L} \mathrm{e}^{-\frac{g_{B}}{\alpha A_{t f f}} P_{i f}\left(1-\mathrm{e}^{-\alpha t}\right)}=P_{i P} \mathrm{e}^{-\alpha L} \mathrm{e}^{-\frac{B_{B}}{A_{t f} L_{\text {eff }}}} \quad \text { using } L_{\text {eff }}=\frac{1-\mathrm{e}^{-\alpha L}}{\alpha}
$$

The output pump power with a zero gain interaction reads simply $P_{P_{o}}(L)=P_{i P} \mathrm{e}^{-\alpha L}$, so that the depletion factor $d$ can be easily derived from Eq.(1) and hence the maximum input signal power $P_{i S}$ for a given tolerable depletion factor $d$ : 


$$
P_{i S}<-\ln (1-d) \frac{A_{e f f}}{g_{B} L_{e f f}} \stackrel{L \rightarrow \infty}{=}-\ln (1-d) \frac{A_{e f f}}{g_{B}} \alpha
$$

This result shows that the maximum CW signal power $P_{i S}$ is totally independent of the power $P_{P}$ and the pulse width of the pump. It only depends on the fiber properties for a given depletion factor $d$.

\section{RELATION BETWEEN DEPLETION AND ERROR ON THE PEAK GAIN FREQUENCY}

Let consider the Brillouin gain spectrum in a short section at the far end of the fiber to be:

$$
g\left(v^{\prime}\right)=g_{B} \frac{1}{1+\left[\left(v^{\prime}-v_{B}\right) / \Delta v / 2\right]^{2}}
$$

where $v_{B}$ is the Brillouin shift corresponding to the maximal gain in the short section and $\Delta v$ the FWHM width of this gain spectrum. To make it simpler, the frequency scale is shifted to $v=v^{\prime}-v_{B}$, (maximum gain at the frequency origin).

If the maximal Brillouin gain frequency in the long uniform segment is shifted by $\delta v$ and the FWHM width $\Delta v$ is identical to the short segment, the pump intensity follows this distribution when the effect of depletion is small $(\mathrm{d}<0.2)$ :

$$
I_{p}(v)=I_{P_{i s}}\left[1-\frac{d}{1+\left(\frac{v-\delta v}{\Delta v / 2}\right)^{2}}\right]
$$

Assuming a very small gain in the percent range during the interaction over the pulse width $T$, the net signal gain can be reasonably expressed by a first order expansion $\left(V_{g}\right.$ : group velocity) of the exponential amplification:

$$
G(v)=1+g(v) I_{P}(v) \frac{V_{g} T}{2}=1+g_{B} \frac{V_{g} T}{2} I_{P_{i s}} \frac{1}{1+\left(\frac{v}{\Delta v / 2}\right)^{2}}\left[1-\frac{d}{1+\left(\frac{v-\delta V}{\Delta v / 2}\right)^{2}}\right]
$$

This distribution is represented in Fig. 1 and presents a maximum that is shifted with respect to the $d=0$ situation. The amount of shifting depends on the magnitude of the depletion $d$ and the relative shift $\delta v$ of the maximum gain frequencies of the two segments. The gain FWHM width $\Delta v$ is just a scaling factor and all results can be expressed as normalized to $\Delta v$. The error is found by calculating $v$ giving the maximum of the expression in Equ.(7). The solution turns out to be the root of a $5^{\text {th }}$ order polynomial that cannot be extracted analytically ${ }^{9}$. Some approximations have to be carried out and a first order solution can be obtained with the assumption that the error is much smaller than $\Delta v$ :

$$
v_{e} \cong-\frac{d \delta v}{\left(1+\left(\frac{\delta v}{\Delta v / 2}\right)^{2}\right)^{2}-d\left(2+\left(\frac{\delta v}{\Delta v / 2}\right)^{2}\right)}
$$

The minus sign indicates the maximum gain is logically shifted to higher frequencies when the long segment presents a peak gain at lower frequencies than in the short segment (as depicted in Fig.1). This expression is maximal when:

$$
\delta v=\Delta v \sqrt{\frac{d+2}{24}} \approx \frac{\Delta v}{3} \text { for a small depletion } d
$$

This result simply means that the error is maximal when the shift between the peaks of the two segments spectra is a third of the full width, while the error vanishes if there is no shift or a shift much larger than the gain spectral width (no more overlap). Inserting (9) into (8) leaves the following inequality:

$$
\left|v_{e}\right|<\left|-\frac{27 d \Delta v}{169-484 d}\right| \approx 0.16|d| \Delta v
$$

An interesting result would be to determine the tolerable amount of depletion that would ensure that the error will never exceed a given value $v_{e}$. Fortunately the $5^{\text {th }}$ order polynomial shows a linear dependence on the parameter $d$, so an exact solution can be extracted. The expression is quite lengthy, but a robust $2^{\text {nd }}$ order approximation is given here:

$$
d_{\max }=\frac{\left(1+e^{2}\right)\left[4 e y^{2}-\left(1+e^{2}\right) y\right]}{3 e y^{2}-\left(2+e^{2}\right) y+e} \quad \text { with } e=\frac{\delta v}{\Delta v / 2} \quad \text { and } \quad y=\frac{v_{e}}{\Delta v / 2}
$$

It must be mentioned that all results shown above equally applies for a loss configuration. In that case a negative value for the depletion factor $d$ has to be inserted in all expressions, leading to similar errors for small $d$. 


\section{DISCUSSION}

A common accuracy claimed in most reports and commercialized instruments is $1 \mathrm{MHz}$ on the peak gain frequency. Assuming a FWHM gain linewidth of $\Delta v=30 \mathrm{MHz}$ and the worst case situation $\delta v=\Delta v / 3=10 \mathrm{MHz}$, we find that the maximum depletion factor must be $d_{\max }=0.17(-0.8 \mathrm{~dB})$ by Eq.11 and as graphed in Fig.2. Inserting this value into Eq.4 and taking realistic values for a standard fiber $\left(A_{\text {eff }}=80 \mu \mathrm{m}^{2}\right.$, $g_{B}=1.510^{-11} \mathrm{~m} / \mathrm{W}, \alpha^{-1}=22 \mathrm{~km}$ ), we can determine that the CW signal power $P_{i s}$ must not exceed $45 \mu \mathrm{W}(-14 \mathrm{dBm})$ to ensure that the depletion never exceeds 0.17 in a very long fiber $\left(L>L_{e f f}=22 \mathrm{~km}\right)$. This value is fairly low and is actually far from being proved to be met in the vast majority of publications and commercial instruments. They are thus all potentially subject to biasing errors related to depletion, errors that are only rarely observed since the worst case situation is very unlikely to occur in real conditions.

In conclusion we have pushed the modeling of the pump depletion effect to obtain analytical expressions that are useful for the proper design of a BOTDA sensor and for the determination of its immunity to the

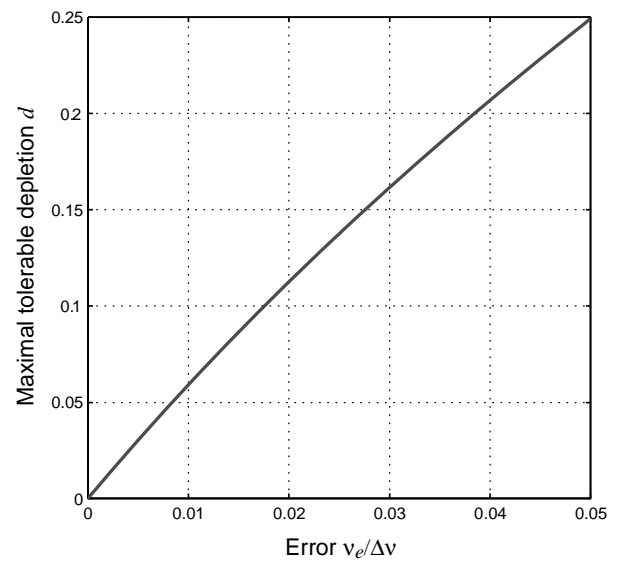

Fig.2: Maximum tolerable depletion $d$ to ensure an error on the determination of the Brillouin shift not exceeding $v_{e}$ in the worst case situation. depletion. This model is informative on the conditions maximizing the depletion effect; therefore a standard configuration can be defined to test the immunity of a set-up to depletion, made of a long uniform fiber having a length equivalent to the claimed range. The Brillouin frequency at the far end of this fiber is shifted by locally modifying the temperature or the applied strain over a short section, albeit longer than the spatial resolution. The amount of shift must be $\delta v=\Delta v / 3$ and the measured Brillouin shift must correspond to the real value that can be obtained by measuring the short segment only.

\section{ACKNOWLEDGEMENTS}

The authors address their warm thanks to Prof. Moshe Tur from Tel Aviv University and to Prof. Miguel Gonzalez Herraez from University of Alcala-Madrid for their critical points of view, crucially helpful for the proper development of this model. This study was realized in the framework of the European COST Action 299 "FIDES".

\section{REFERENCES}

[1] T. Horiguchi and M. Tateda, "Optical-fiber-attenuation investigation using stimulated Brillouin scattering between a pulse and a continuous wave," Opt. Lett. 14(8), 408-410 (1989).

[2] L. Thévenaz, "Brillouin distributed time-domain sensing in optical fibers: state of the art and perspectives," Frontiers of Optoelectronics in China 3(1), 13-21 (2010).

[3] M. A. Soto, G. Bolognini, F. Di Pasquale and L. Thévenaz, "Long-range Brillouin optical time-domain analysis sensor employing pulse coding techniques," Measurement Science and Technology 21(9), 094024 (2010).

[4] H. Liang, W. Li, N. Linze, L. Chen and X. Bao, "High-resolution DPP-BOTDA over 50 km LEAF using return-to-zero coded pulses," Opt. Lett. 35(10), 1503-1505 (2010)

[5] S. Martin-Lopez, M. Alcon-Camas, F. Rodriguez, P. Corredera, J. D. Ania-Castañon, L. Thévenaz and M. Gonzalez-Herraez, "Brillouin optical time-domain analysis assisted by second-order Raman amplification," Opt. Express 18(18), 18769-18778 (2010).

[6] A. Fellay, L. Thévenaz, M. Facchini and P. A. Robert, "Limitation of Brillouin time-domain analysis by Raman scattering," in 5th Optical Fibre Measurement Conference C. Boisrobert, Ed., pp. 110-113, Université de Nantes, Nantes, France (1999)..

[7] D. Alasia, M. Gonzalez Herraez, L. Abrardi, S. Martin-Lopez and L. Thevenaz, "Detrimental effect of modulation instability on distributed optical fiber sensors using stimulated Brillouin scattering," in 17th International Conference on Optical Fibre Sensors M. Voet, R. Willsch, W. Ecke, J. Jones and B. Culshaw, Eds., pp. 587-590, SPIE, Bruges, Belgium (2005)..

[8] T. Horiguchi, K. Shimizu, T. Kurashima, M. Tateda and Y. Koyamada, "Development of a distributed sensing technique using Brillouin scattering," Journal of Lightwave Technology 13(7), 1296-1302 (1995).

[9] E. Geinitz, S. Jetschke, U. Röpke, S. Schröter, R. Willsch and H. Bartelt, "The influence of pulse amplification on distributed fibre-optic Brillouin sensing and a method to compensate for systematic errors," Measurement Science and Technology 10(2), 112 (1999).

[10] A. Minardo, R. Bernini, L. Zeni, L. Thevenaz and F. Briffod, "A reconstruction technique for long-range stimulated Brillouin scattering distributed fibre-optic sensors: Experimental results," Measurement Science \&amp; Technology 16(4), 900-908 (2005).

[11] Y. Dong, L. Chen and X. Bao, "System optimization of a long-range Brillouin-loss-based distributed fiber sensor," Appl. Opt. 49(27), 5020-5025 (2010). 